\title{
Optimization of Dual Motion Mechanism with Double Grooved Cams for High-voltage Gas Circuit Breaker
}

Masanao Terada ${ }^{1,2, *}$, Yuki Nakai ${ }^{3}$, Hiroaki Hashimoto ${ }^{1}$, Daisuke Ebisawa ${ }^{3}$, Hajime Urai $^{1}$, Yasunobu Yokomizu ${ }^{2}$

${ }^{1}$ Research \& Development Group, Hitachi, Ltd., 319-1292, Japan

${ }^{2}$ Department of Electrical Engineering, Nagoya University, 464-8603, Japan

${ }^{3}$ Energy Business Unit, Hitachi, Ltd., 316-8501, Japan

\begin{tabular}{l} 
A R T I C L E I N F O \\
\hline Article history: \\
Received: 02 April, 2020 \\
Accepted: 19 June, 2020 \\
Online: 12 July, 2020
\end{tabular}

Keywords:

Circuit Breaker

Capacitive Current Switching

Dual Motion

Grooved Cam

\begin{abstract}
A B S T R A C T
A novel design of a dual motion mechanism for a high-voltage gas circuit breaker is presented. The contact motion of the circuit breaker due to the operating mechanism increases capacitive current switching (CCS) performance. CCS is one of the interrupting duties of the circuit breaker, where high voltage is applied during the half cycle from contact separation. The dual motion mechanism drives two contacts in opposite directions from each other. Operating energy is reduced because the maximum displacement of the moving parts linked to the operating mechanism is shortened. To increase CCS performance at lower operating energies, the contact on the opposite side of the contact linked to the operating mechanism requires quick motion in the CCS period with a short displacement. The dual motion mechanism reported here is composed of two grooved cams that cross each other (double grooved cams). A pin positioned at the intersection point of the grooved cams rotates a lever linked to both contacts while changing the lever ratio that shortens the path length of the pin movement. To implement an optimized displacement curve with low operating energy and low mechanical stress while keeping the CCS performance high, a shape optimization method was developed that uses a direct search to minimize the local contact forces acting on the contact positions between the grooved cams and the pin. In order to maintain the stability of the pin in motion, a position holding part was designed by considering size of the gaps between the grooved cams and the pin. The measured displacement curve was in good agreement with the ideal one. In addition, a fullscale prototype was fabricated that successfully passed a 10,000-motion test.
\end{abstract}

\section{Introduction}

This paper is an extension of the work originally presented at the 2019 5th International Conference on Electric Power Equipment - Switching Technology (ICEPE-ST) [1]. The changes from the conference paper are as follows. First, in section 2, the role of a high-voltage gas circuit breaker is described in Figure 1 to clarify the relationship with the target motion mechanism. Second, in section 3, the design method of the displacement curve is described in Figure 4. The method aims at achieving reductions in the operating energy and mechanical load while maintaining high capacitive current switching (CCS) performance as is required for the high-voltage gas circuit breaker. An optimal

*Corresponding Author: Masanao Terada, Email: masanao.terada.br@hitachi.com solution is determined from the trade-off relationship between the operating energy and the mechanical load, as shown in Figure 8. Third, section 4 describes the method for optimizing the shape of the double grooved cams in detail by referring to the flowchart shown in Figure 9 and the position-holding parts for stabilizing the movements of the cams shown in Figure 14. In addition, the critical intersection angle of the double grooved cams is described in Figure 13. Furthermore, a comparison of optimization results for two different initial configurations and an analysis of the convergence process of the optimal calculation are given in Figure 15. Finally, section 5 describes the method used to verify the operation of the double grooved cams in Figure 17.

This paper contributes to design studies of general motion mechanisms by proposing the motion mechanism suitable for the 


\section{Terada et al. / Advances in Science, Technology and Engineering Systems Journal 5, No. 4, 109-118 (2020)}

gas circuit breaker, optimizing the displacement curve that contributes to low operating energy, and providing the shape optimization method of the motion mechanism.

High-voltage circuit breakers require low operating energy for the sake of high reliability and low cost. Several manufacturers have proposed motion mechanisms to increase separation speed of contact with low operating energy [2-4]. Reference [2] describes a double-speed mechanism which moves a movable-side contact linked to the operating mechanism quickly with an auxiliary cam. Moreover, reference $[3,4]$ describes dual motion systems that drive two contacts in opposite directions from each other. The dual motion mechanisms can shorten the moving distance of the movable-side (drive-side) contact, which speeds up the heavy drive-side moving parts. By driving a light contact on the opposite side (driven-side) parts, which is fixed in the conventional single motion system, the relative speed between the driven-side contact and drive-side contact becomes sufficiently high with lower operating energy.

CCS is one of the interrupting duties, which requires withstanding of a recovery voltage to be applied during a halfcycle from contact separation. In particular, CCS requires a high gas density inside the insulation nozzle that covers the driven-side contact and a low electric field between the contacts. To increase the gas density between contacts, the density drop inside the nozzle due to flow acceleration caused by the supersonic flow should be suppressed [5-11]. To decrease the electric field between the contacts, their separation in the period of the half-cycle from contact separation has to be increased; that is, the contacts must move at a fast speed. Reference [12] shows the optimal opening speeds for CCS depending on rated voltage. Reference [13] indicates that faster motions resulting in a reduced electric field and a longer nozzle throat length that avoids a density drop are effective ways to increase CCS performance. The first goal of this paper is to present the optimal motion properties for increasing CCS performance and the second one is to describe a motion mechanism that realizes these properties, presupposing a nozzle with a sufficiently narrow cross-sectional area between the inner nozzle wall and the driven-side contact so as not to affect the density drop.

To optimize the displacement curve for higher CCS performance, it is important to control the speed at every moment and the timing when the driven-side contact starts to move. Reference [14] shows that CCS performance is improved when the driven-side contact starts to move $3 \mathrm{~ms}$ before contact separation. Moreover, if the CCS performance with the optimum displacement curve is the same as that of the initial displacement curve, the operating energy with the optimum displacement curve is smaller than that with the initial displacement curve. To ensure reliability, it is also important to reduce mechanical stress. The higher the speeds of the contacts are, the higher the load acting on the dual motion mechanism becomes. This requires that the acceleration be limited at the beginning and the end of the motion. Therefore, it is desirable for a motion mechanism to be able to express any displacement curve. Accordingly, a new type of dual motion mechanism is proposed, which is composed of two arbitrarily shaped grooved cams crossing each other. A cam design method using an arbitrarily shaped spline function has been proposed [15]. Reference [16] applied this cam design method to a rotating cam of an operating mechanism. The idea for the motion mechanism involving crossing several grooved cams comes from other fields and has been embodied in refuelling devices [17].

This paper describes the design of a displacement curve on the driven-side of the high-voltage circuit breaker in a way that minimizes the operating energy and mechanical load acting on the mechanism while keeping CCS performance high. Besides, it describes an optimal design and a mounting structure for a dual motion mechanism with double grooved cams. Furthermore, the operation of the mechanism is verified with a small-sized test setup and a real-scaled prototype of the high-voltage circuit breaker.

\section{Proposed Dual Motion Mechanism}

Figure 1 shows the high-voltage gas circuit breaker with the dual motion mechanism. During normal operation in electric power transmission, the main drive-side and driven-side contacts are in contact with each other to conduct the normal load current. Upon interrupting a short circuit current, the drive-side main contact linked to the operating mechanism is pulled in the opening direction, and the contacts separate. Briefly, after the main contact separation, the arcing contacts separate. At this time, an arc discharge is generated between the arcing contacts. The arc is extinguished by gas blowing due to the pressure rise resulting from mechanical compression and heating by an arc in the pressure build-up chamber. The dual motion mechanism drives the drivenside arcing contact in the opposite direction of the driving direction of the drive-side arcing contact. If the relative speeds of the driveside and driven-side contacts are similar to the speed of the driveside arcing contact only, the operating energy can be lowered and the cost of the operating mechanism can be reduced because of the reduction in the drive-side speed.

Figure 2 shows schematic drawings of the proposed dual motion mechanism and its motion. The displacement curves of the motion in Figures 2 (a)-(d) are shown in Figure 3. The operating mechanism pulls a drive rod with a grooved cam (the movable grooved cam). Until the drive pin reaches the curve of the movable grooved cam, in the period between the beginning of the drive-side motion and timing (a), the lever does not rotate because the drive pin does not apply any driving force to the lever. Therefore, the driven rod is motionless. When the curved surface of the movable grooved cam comes into contact with the drive pin, the lever rotates and the driven rod starts to move. While the drive pin moves along the stationary grooved cam, which is cut into a guide plate, the lever continues to rotate, changing the lever ratio of the driven-side arm length to drive-side arm length. When the drive pin reaches the end of the stationary grooved cam, the motion of the driven rod is finished because the lever stops rotating. After timing (d), only the drive rod moves because the drive pin does not apply any driving force to the lever.

This dual motion mechanism with double grooved cams has three advantages. The first one is that an arbitrarily shaped displacement curve can be expressed. The second one is that the driving force of the drive pin can be converted efficiently into rotational force of the lever because the drive pin's moving direction matches the vertical direction of the lever for adjusting the shape of the cam curves. The third one is that the overall size of the dual motion mechanism can be reduced because the path 
length of the pin movement is shortened due to the lever ratio changing during the lever rotation.

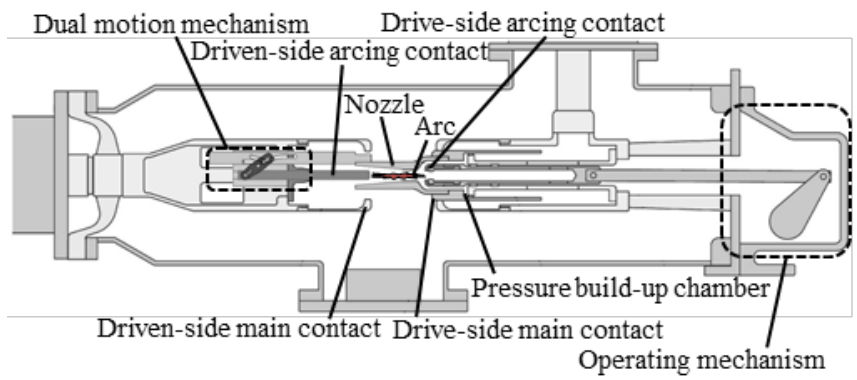

Figure 1: Overview of high-voltage gas circuit breaker with dual motion

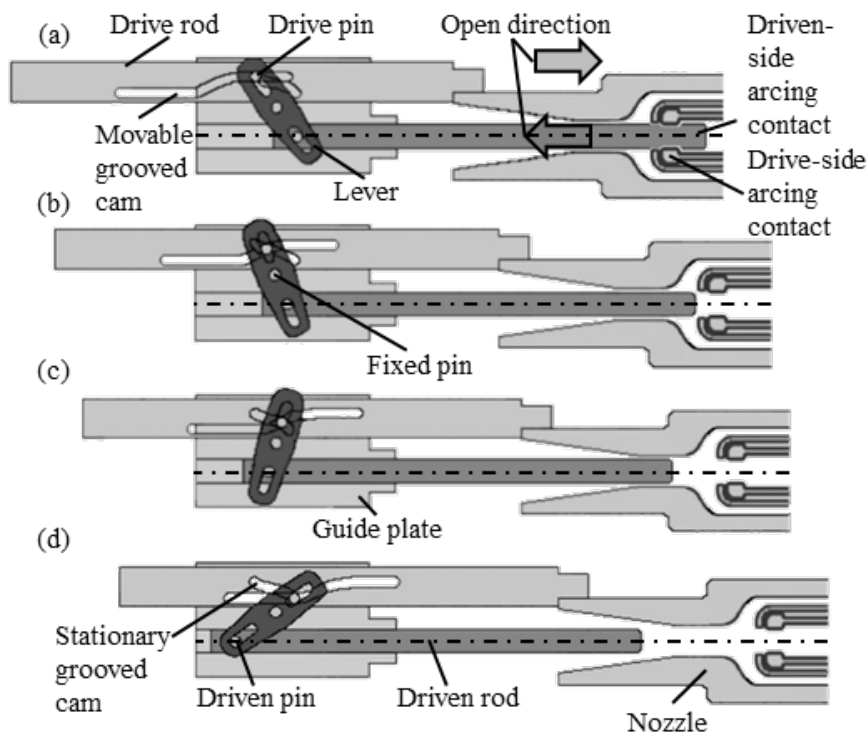

Figure 2: Schematics of the proposed dual motion mechanism

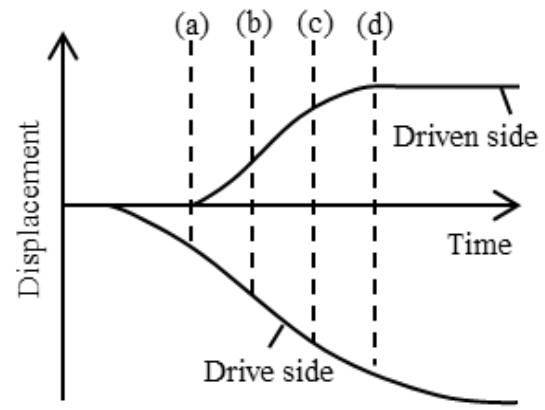

Figure 3: Drive-side and driven-side Displacement curves

\section{Optimization of Driven-side Displacement Curve}

To minimize the operating energy and reduce the mechanical load while keeping CCS performance high, it is desirable for optimizing the driven-side displacement curve.

\subsection{Method of Designing Driven-side Displacement Curve}

The steps in the design method for the driven-side displacement curve is shown in the flowchart of Figure 4 and described in detail here.

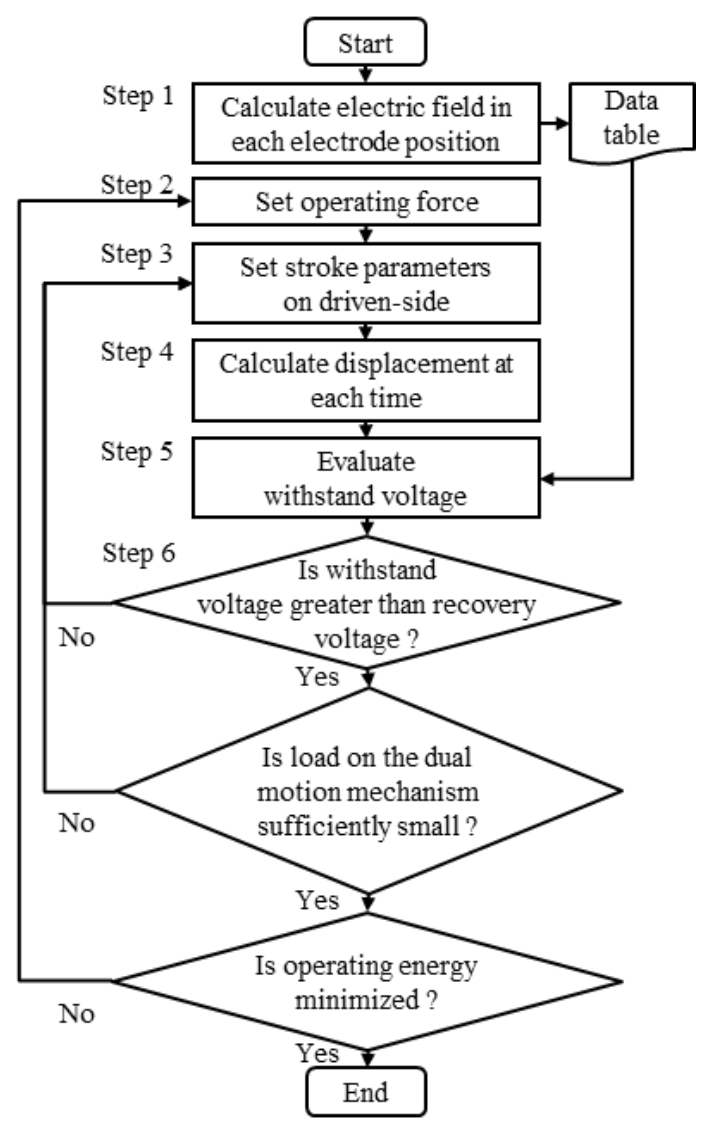

Figure 4: Design flow of displacement curve

Step 1: Calculate the electric field with the specified pitch from the position of the contact separation to the position of the halfcycle of applied voltage with power frequency, based on the electrode shape needed to withstand the lightning impulse voltage in the open position. The calculation results are saved as a data table.

Step 2: Set the operating force of the operating mechanism, which is defined by a spring force at the start position of the drive-side motion. The operating energy is calculated from the spring forces at the beginning and the end of the drive-side motion and the drive-side maximum displacement. The operating energy is proportional to the operating force which is calculated with the spring constant.

Step 3: Set the parameters of the displacement curve on the driven side in Figure 5 (a). Here, an intermittent motion with straight sliding parts of the movable grooved cam enables the start timing of the driven-side motion to be adjusted. The start timing and the end timing of the driven-side motion are the first and $n$-th motion points, respectively. The start timing and the end timing of the drive-side motion are the 0 -th and $(n+1)$-th motion points, respectively. To set an arbitrarily time-varying displacement curve, the ratio of the speed of the driven side to that of the drive side is expressed with a Catmull-Rom spline of degree 3 [18] in Figure 5 (b). The control points of the spline curve are the design parameters. The speed ratio $l(t)_{j}$ between the $j$-th and $(n+1)$-th control points with the Catmull-Rom spline curve is expressed as follows, 


$$
\begin{aligned}
l(t)_{j}=\left(-\frac{1}{2} l_{j-1}\right. & \left.+\frac{3}{2} l_{j}-\frac{3}{2} l_{j+1}+\frac{1}{2} l_{j+2}\right) t^{3} \\
& +\left(l_{j-1}-\frac{5}{2} l_{j}+\frac{5}{2} l_{j+1}+\frac{1}{2} l_{j+2}\right) t^{2} \\
& +\left(-\frac{1}{2} l_{j-1}+\frac{1}{2} l_{j+1}\right) t+l_{j},
\end{aligned}
$$

where $l_{j}$ is the $j$-th control point of the speed ratio and $t$ is a real number in the range from 0 to 1 . When the width of the drive-side displacement is $\Delta X_{d}$, the $i$-th speed ratio $L_{i}$ between $l_{4}$ and $l_{5}$ in Figure 5 (c) can be expressed as follows,

$L_{i}$

$=\alpha \frac{\left(x\left(t_{k+1}\right)_{4}-X_{i}\right) l\left(t_{k}\right)_{4}+\left(X_{i}-x\left(t_{k}\right)_{4}\right) l\left(t_{k+1}\right)_{4}}{x\left(t_{k+1}\right)_{4}-x\left(t_{k}\right)_{4}}$,

where $X_{i}$ is the $i$-th drive-side displacement, $x\left(t_{k}\right)_{4}$ is the $k$-th drive-side displacement between $x_{4}$ and $x_{5}, l\left(t_{k}\right)_{4}$ is the $k$-th speed ratio between $l_{4}$ and $l_{5} . \alpha$ is a coefficient to adjust the total amount of driven-side displacement, which is divided by the sum of $L$ from the starting to the end of the displacement. The expression for $\alpha$ is

$$
\alpha=\frac{X_{d n, n}}{\sum_{i=1}^{n} L_{i} \Delta X_{d}} .
$$

Step 4: Evaluate the time-variant displacement on the drive side and driven side given by the operating force $F$ from the law of conservation of energy,

$$
\frac{1}{2} M_{d} V_{d}{ }^{2}+\frac{1}{2} M_{d n} V_{d n}{ }^{2}=\int F \cdot V_{d} d t,
$$

where $V_{d}$ and $V_{d n}$ are the drive-side speed and driven-side speed, respectively. $M_{d}$ and $M_{d n}$ are the drive-side mass and driven-side mass, respectively. $V_{d n}$ is the product of $V_{d}$ and $L$. The load acting on the dual motion mechanism is calculated from the driven-side mass and the acceleration obtained from Equation (4).

Step 5: Evaluate the withstand voltage $V_{B D}$ at each contact position with the displacements of both sides obtained from Step 3 and the electric field at each position with the data table made in Step 1. $V_{B D}$ is calculated using Equation (5) [19],

$$
V_{B D}=100 \cdot \frac{A P^{n}}{E_{0}},
$$

where $E(\% / \mathrm{mm})$ is the calculated value, $P(\mathrm{MPa})$ is absolute pressure, and $A$ and $n$ are coefficients that vary in accordance with the gas condition and the shape of the gas circuit breaker.

Step 6: Optimize the parameters of the displacement curve on the driven side until the withstand voltage is greater than the recovery voltage and the load acting on the dual motion mechanism is sufficiently small.

Step 7: Minimize the operating force.

\subsection{Design Example of Displacement Curve}

The design parameters are the controlled points of the speed curve on the driven side. The calculation conditions are as follows:

- Frequency: $60 \mathrm{~Hz}$

- Gas pressure: $0.6 \mathrm{MPa}$

- Maximum displacement ratio of driven- to drive-side: 0.28 www.astesi.com

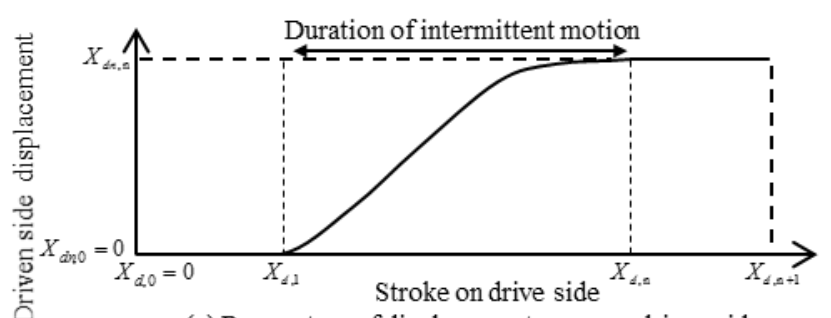

(a) Parameters of displacement curve on driven side
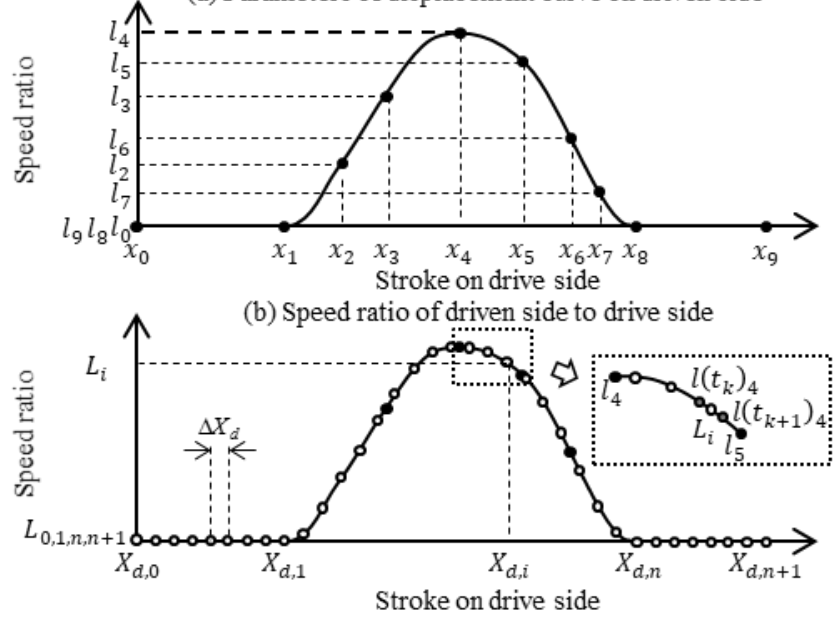

(c) Speed ratio divided by a small width

Figure 5: Expression of driven-side displacement curve

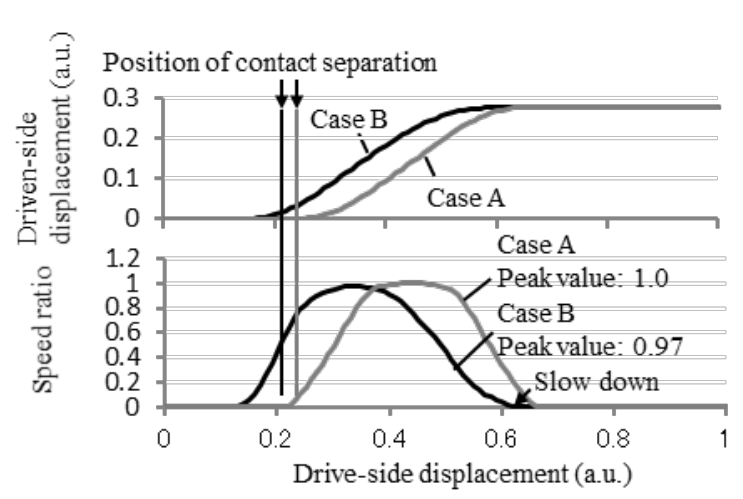

Figure 6: Input conditions for case A and case B: displacement curve (upper graph) and speed ratio (lower graph).

Figure 6 shows the input conditions for case A and case B. In case A, the movement starts at the same time as contact separation. In case $\mathrm{B}$, the movement starts before contact separation, and the maximum speed ratio and acceleration in the latter half of the driven-side displacement decrease in comparison with case A. Figure 7 shows the results of the calculation. The operating energy in case B is lower than that in case A. The withstand voltages against recovery voltage for $\mathrm{CCS}$ in case $\mathrm{A}$ and case $\mathrm{B}$ are similar. This is because the driven-side speed at contact separation in case $\mathrm{B}$ is higher than in case A with the same operating energy. The mechanical load acting on the driven side in case B is lower than in case A because of the lower operating energy. To show that case $\mathrm{B}$ is the optimum solution, Figure 8 illustrates the relationship between the operating energy and the mechanical load. The drivenside speeds of the plotted squares are 1.2 times and 1.5 times faster than in case $\mathrm{A}$, with the condition of the same timing of the motion. As the driven-side speed increases, the operating energy decreases 
to have the same CCS performance. However, the mechanical load increases. In contrast, both operating energy and mechanical load are compatibly low in case B.

An optimized solution to improve CCS performance can be obtained by adjusting the driven-side displacement. The drivenside displacement of case B is assumed in section 4 to describe the design of the dual motion mechanism with the double grooved cams.
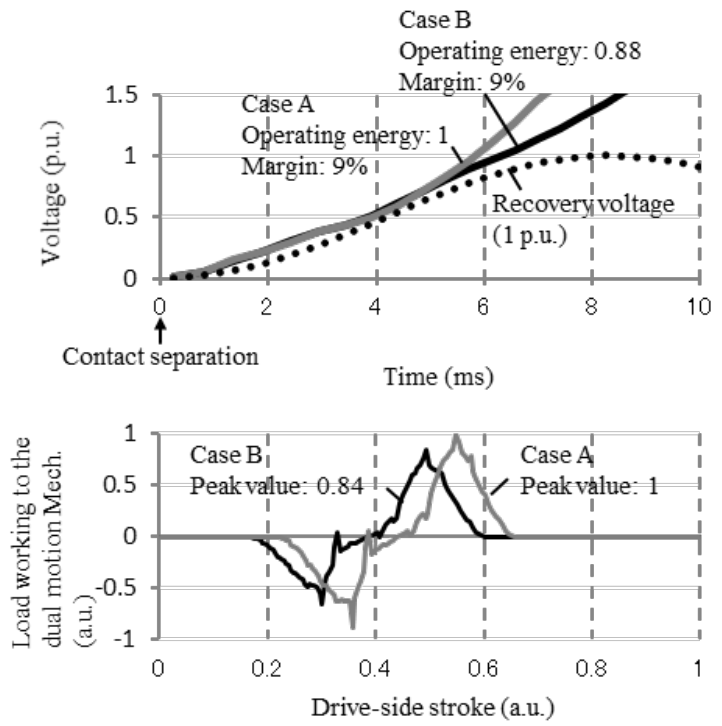

Figure 7: Results of calculation for case A and case B. The upper graph shows withstand voltages for CCS. The lower graph shows mechanical loads acting on the dual motion mechanism.

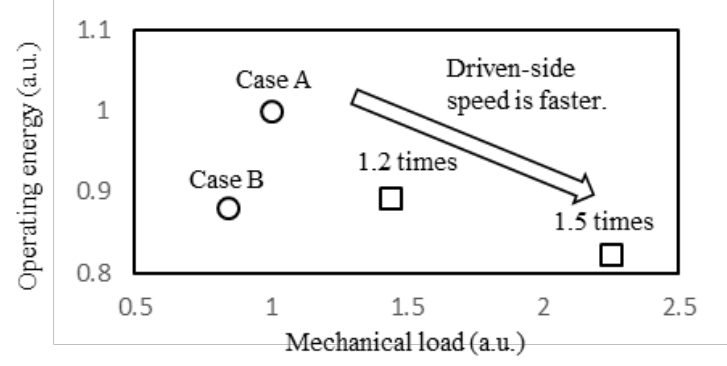

Figure 8: Relationship between operating energy and mechanical load acting on the dual motion mechanism

\section{Shape Optimization of Double Grooved Cams}

\subsection{Method of designing the shape of double grooved cams}

The dual motion mechanism with double grooved cams can be easily implemented in any shape because they have a high degree of design freedom. On the other hand, it is important to optimize the shape of the grooves because the motion mechanism must be compact and it must have mechanical strength and stability. In the following, the design method of the double grooved cams is described by referring to the flowchart shown in Figure 9.

Step 1: Input the drive-side and driven-side displacements shown in Figure 5.

Step 2: Set the dimensions for the dual motion mechanism with double grooved cams, which are the drive-side initial arm length of the link lever $L_{d, 0}$, the initial lever angle $\theta_{0}$, the driven-side arm

www.astesj.com length of the link lever $L_{d n}$, and the grooved cam width $W$ shown in Figure 10. The end points $P_{0}$ and $P_{m}$ of the stationary grooved cam are determined from these structural parameters and the driven-side maximum displacement $X_{d n, n}$.

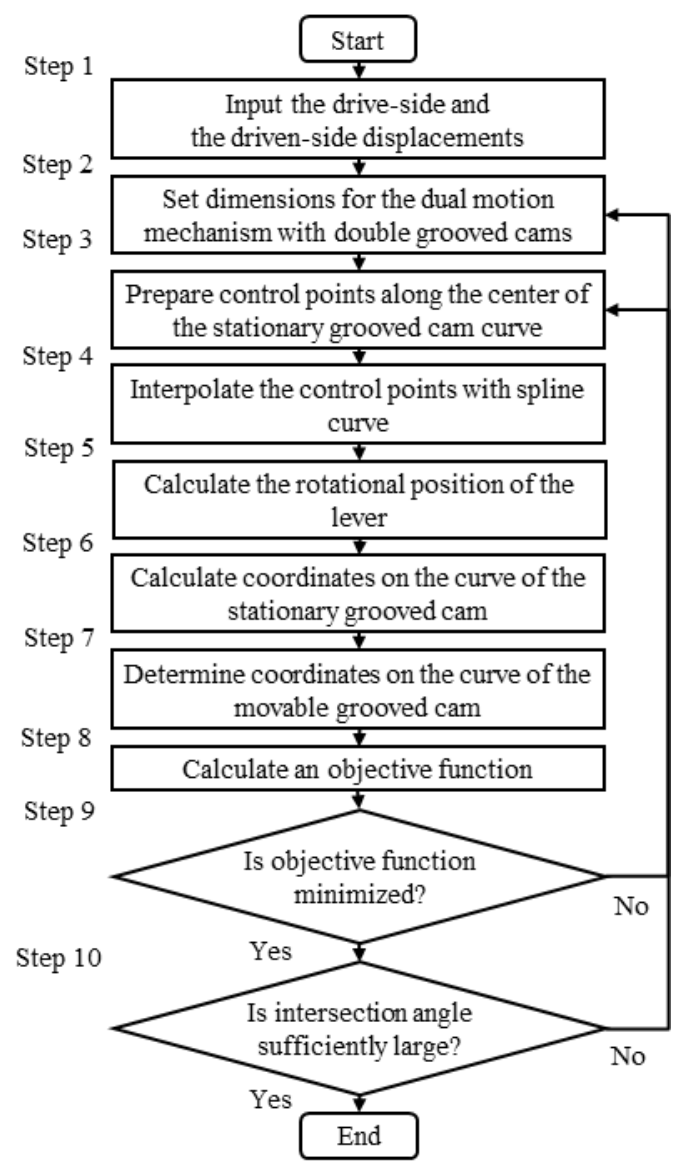

Figure 9: Design flow for shape of the dual motion mechanism with double grooved cams

Step 3: Prepare control points along the center line of the stationary grooved cam curve. $P_{i}$ is the $i$-th control point of the stationary grooved cam $(0 \leq i \leq m)$. To smooth the shape of the stationary grooved cam, the shape of the curve from $P_{0}$ to $P_{m}$ must be a monotonic function. Therefore, the constraints between two neighbouring points are $P_{i, x}>P_{i+1, x}$ and $P_{i, y}>$ $P_{i+1, y}$, where $P_{i, x}$ is the $x$-component of $P_{i}$ and $P_{i, y}$ is the $y$ component of $P_{i}$.

Step 4: Interpolate the control points with a Catmull-Rom spline of degree 3. The point $P$ between $P_{i}$ and $P_{i+1}$ is described in accordance with Equation (1), using $P_{i-1}, P_{i}, P_{i+1}$ $P_{i+2}$ and $t$ which is a real number in the range of 0 to 1 .

Step 5: Calculate the rotational positions of the link lever on the driven-side $Q_{d n, j}$, the rotational angle $\theta_{j}$, and the drivenside displacement $X_{d n, j}$, shown on the left side of Figure 11 by using Equations (6) and (7),

$$
\begin{gathered}
Q_{d n, j, x}=S_{d n} \sin \theta_{0}-X_{d n, j}, \\
Q_{d n, j, y}=-S_{d n} \cos \theta_{j},
\end{gathered}
$$

where $\theta_{j}$ is expressed as 


$$
\theta_{j}=\arcsin \left(\frac{S_{d n} \cos \theta_{0}-X_{d n, j}}{S_{d n}}\right) .
$$

Step 6: Calculate the intersection point of the link lever and the stationary grooved cam $Q_{f, j}$ in accordance with the rotational points $Q_{d n, j}$, as shown on the right side of Figure 11. $Q_{f, j}$ is described using the k-th point $P\left(t_{k}\right)_{i}$ and $\mathrm{k}+1$-th point $P\left(t_{k+1}\right)_{i}$ between the control points $P_{i}$ and $P_{i+1}$ and a parameter $\alpha$ as follows,

$$
\begin{aligned}
& Q_{f, j}=\alpha\left\{P\left(t_{k+1}\right)_{i}-P\left(t_{k}\right)_{i}\right\}+P\left(t_{k}\right)_{i}, \\
& \alpha \\
& =\frac{P\left(t_{k+1}\right)_{i, x}-P\left(t_{k}\right)_{i, x}}{-P\left(t_{k}\right)_{i, x}+\tan \theta_{j} P\left(t_{k}\right)_{i, y}} \\
& -\tan \theta_{j} \frac{P\left(t_{k+1}\right)_{i, y}-P\left(t_{k}\right)_{i, y}}{-P\left(t_{k}\right)_{i, x}+\tan \theta_{j} P\left(t_{k}\right)_{i, y}} .
\end{aligned}
$$

Step 7: Determine the intersection point of the stationary grooved cam and the intersection point of the movable grooved cam $Q_{d, j}$, which is expressed as

$$
Q_{d, j}=Q_{f, j}-X_{d, j}
$$

Step 8: Calculate an objective function to minimize the contact forces. In this dual motion mechanism, the drive pin is inserted at the intersection point of the stationary grooved cam and the movable grooved cam shifts while the operating mechanism pulls the movable grooved cam. Therefore, contact forces act at the points of contact between the drive pin and the surface of the grooved cams. For mechanical reliability, it is desirable for the contact force to be low. This mechanism has two possible ways to increase the contact force. The first is that the intersection angle between the double grooved cams becomes too small. The smaller the intersection angle is, the larger the contact force becomes, resulting in the possibility of a pin lock occurring during operation. This is called the wedge effect, as explained in section 4.2. The second possibility is that the contact stress becomes too large. The smaller the local curvature radius is, the larger the contact stress becomes because of the decreasing contact area. The intersection angle $\varphi$ is determined to be the acute angle between the tangential line of the stationary grooved cam and the tangential line of the movable grooved cam. The local curvature radius $R_{s}$ of the stationary grooved cam is determined as the radius of a circle calculated from three adjacent control points. The local curvature radius $R_{m}$ is determined in the same way. $\varphi, R_{s}$, and $R_{m}$ are shown in Figure 12. The dimensionless objective function $O$ is expressed as

$$
\begin{aligned}
O=G_{1}\left(\frac{\varphi_{t}}{\min \varphi}\right) & +G_{2}\left(\frac{R_{t}}{\min R_{f}}\right) \\
& +G_{2}\left(\frac{R_{t}}{\min R_{m}}\right),
\end{aligned}
$$

where $\varphi_{t}$ and $R_{t}$ are criteria and $G_{1}$ and $G_{2}$ are weight coefficients. It is desirable for $G_{1} \gg G_{2}$ because a small intersection angle has the severe effect of increasing the contact force. The design described in section 4.3 sets $G_{1}$ to 100 and $G_{2}$ to 1 . In addition, $G_{1}$ is set to zero if the intersection angle is larger than the criterion. The criterion of the intersection angle is also evaluated in section 4.2.

Step 9: Iterate the procedures of Step 3 to Step 7 to minimize the objective function. The design variables are the $x$ and $y$ coordinates of the control points $P$ on the stationary grooved cam. The Rosenbrock method [20] is used for optimization, which is a type of direct search. This method leads quickly to a convergent and stable solution, but there is a possibility that a local minimum solution is reached. To avoid a local minimum solution, the best one is selected from several solutions in accordance with several initial conditions.

Step 10: Perform the calculation by changing the initial dimensions if the criterion of the intersection angle is not exceeded.

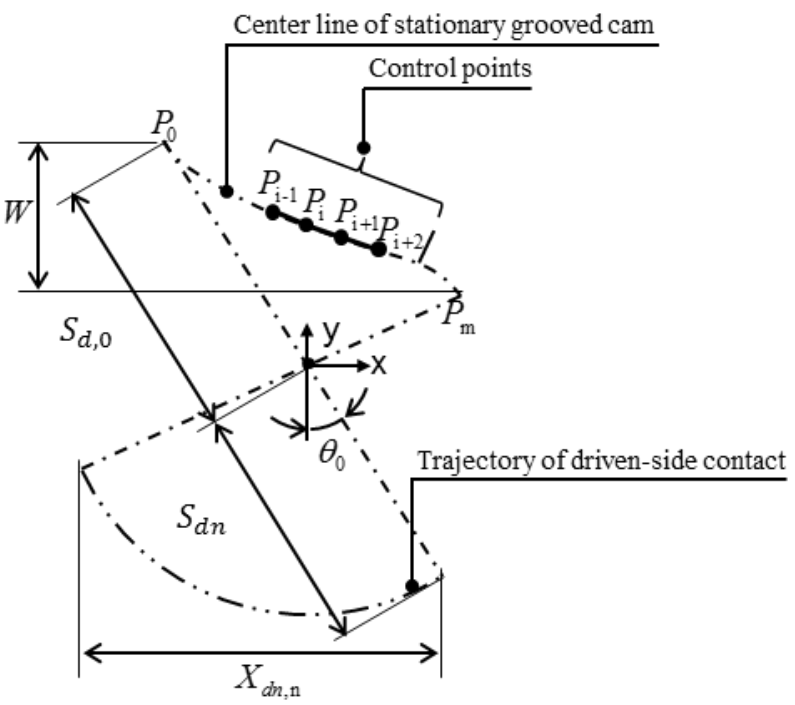

Figure 10: Structural parameters and control points for stationary grooved cam

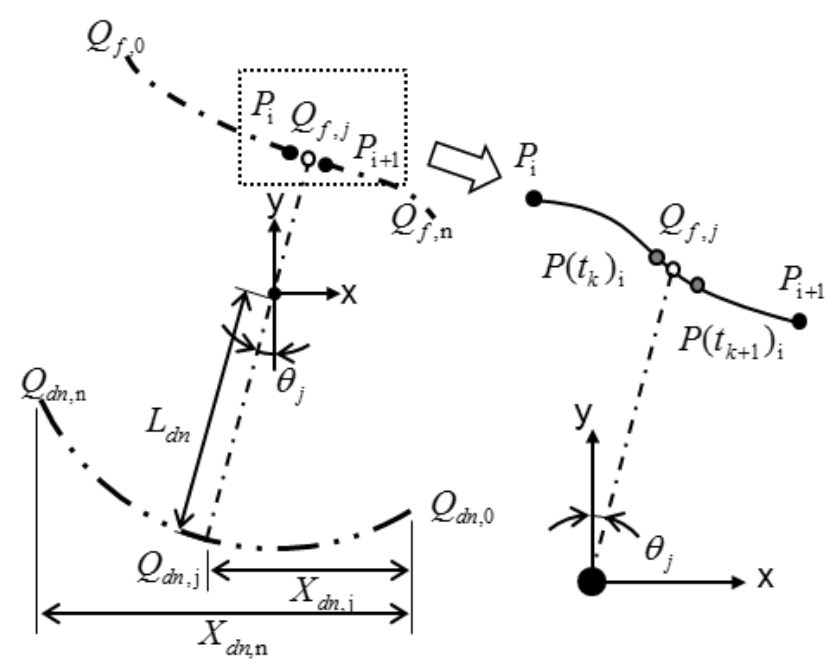

Figure 11: Moving positions on driven side and angles

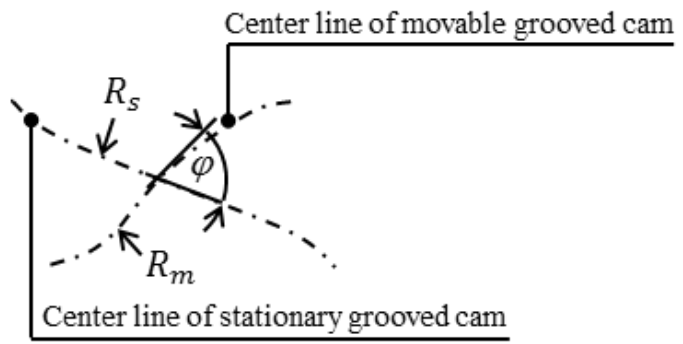

Figure 12: Intersection angle and curvature radii 


\subsection{Criterion of intersection angle between grooved cams}

To design reliable double grooved cams, a criterion for the intersection angle of the stationary grooved cam and the movable grooved cam has to be established. The contact positions of the grooved cams and the drive pin generate nominal forces. The smaller the intersection angle, the larger the friction force induced by the nominal force. A large friction results in a pin lock due to the wedge effect during operation. This requires that the intersection angle be larger than a certain value.

Figure 13 defines the nominal forces and friction forces around the drive pin. When the lever (not illustrated) pushes the drive pin, the friction force acts on the contact position between the drive pin and the movable grooved cam. When the lever pushes the drive pin with a force $F_{0}$ (smaller than the friction force), which is the nonslip condition, the drive pin presses the upper surface of the stationary grooved cam with a force $F_{0}{ }^{\prime}\left(=F_{0}\right)$. Therefore, a nominal force $F_{1}{ }^{\prime} \quad\left(=F_{1}\right)$ acts on the contact position between the drive pin and the stationary grooved cam, as described by the following equation,

$$
F_{1}=\frac{F_{0}}{\sin \varphi}
$$

Furthermore, a nominal force $F_{2}{ }^{\prime}\left(=F_{2}\right)$ acts on the lower surface of the movable grooved cam,

$$
F_{2}=\frac{F_{0}}{\sin \varphi} \cos \varphi=\frac{F_{0}}{\tan \varphi} .
$$

The friction force $F_{3}$ is

$$
F_{3}=\mu F_{2}{ }^{\prime}=\mu \frac{F_{0}}{\tan \varphi},
$$

where $\mu$ is the friction coefficient. If $F_{3}>F_{0}$, pin lock occurs during operation. Therefore, the locking condition is:

$$
\tan \varphi<\mu \text {. }
$$

If $\mu$ is $0.3, \varphi$ must be more than 17 degrees. The criterion $\varphi_{t}$ is decided with some margin for mechanical reliability.

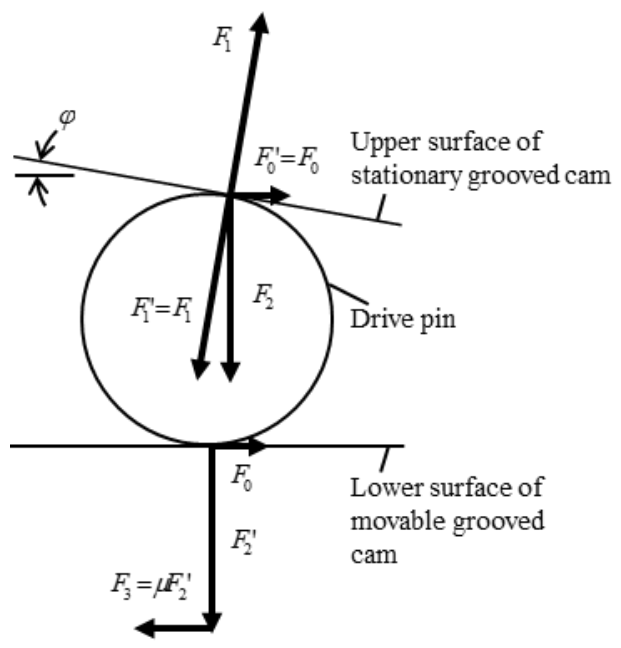

Figure 13: Contact forces and nominal forces on each contact surface

\subsection{Method of structural design of double grooved cams}

A method for designing the pin position holding part is presented for stabilizing the dual motion of the cam mechanism described in section 4.1. Figure 14 is a schematic drawing for distortion of the lever position caused by inclination of the drive pin. Pin rotation around the vertical axis in relation to the front view of Figure 14 caused by backlash in the grooved cams is considered. The inclination of the drive pin is defined by $\delta$. A small inclination of the drive pin occurs due to backlash between the other pins and holes. However, when $\delta$ is greater than the backlash described above, a force resulting from the inclination of the drive pin is transmitted to the lever. Accordingly, the torque between the drive pin and the lever distorts the fixed pin and the driven pin and increases their stress. Finally, when the stress greatly exceeds the yield point, the pins deform plastically, resulting in destruction of the parts of the mechanism. Therefore, the inclination of the drive pin must be reduced. To reduce the inclination, pin holding parts are assembled at both ends of the pin. Even if the drive pin is inclined, the mechanism will not be destroyed because the pin holding parts touch the surface of the lever and force it to an ordinary position. $\delta$ and the distortion between the pin holding part and the surface of the lever $\Delta L_{p}$ are described as

$$
\begin{gathered}
\delta=L_{p} \sin \psi, \\
\Delta L_{p}=\frac{L_{p}}{2}(1-\cos \psi)+\frac{D_{h}}{2} \sin \psi,
\end{gathered}
$$

where $\psi$ is the drive-pin inclination angle, $D_{h}$ is the outer diameter of the pin holding part, and $L_{p}$ is the distance between the ends of the pin holding parts. Once $\psi$ is derived from Equations (17) and (18), $\Delta L_{p}$ is described as follows:

$$
\Delta L_{p}=\frac{L_{p}}{2}-\sqrt{\left(\frac{L_{p}}{2}\right)^{2}-\left(\frac{\delta}{2}\right)^{2}}+\frac{D_{h} \delta}{2 L_{p}} .
$$

The threshold of the inclination of the drive pin $\delta_{t}$ is

$$
\delta_{t}=\delta_{f}+\frac{L_{d}}{L_{d n}}\left(\delta_{f}+\delta_{n}\right),
$$

where $\delta_{f}$ and $\delta_{n}$ are inclinations of the fixed pin and the driven pin, respectively. $L_{d}$ is the distance between the centers of the fixed pin and the drive pin. $L_{d n}$ is the distance between the centers of the fixed pin and the driven pin. From Equation (19), the dimension $D_{h}$ is determined to be a range such that $\delta$ is less than $\delta_{t}$

\subsection{Design Example of Double Grooved Cams}

The double grooved cams were designed to mitigate the contact forces acting on the contact positions between the grooved cams and the drive pin while keeping the overall size as small as possible so that the decided driven-side displacement curve in case B of Figure 6 was realized. Table 1 shows the design examples. Case $I$ is the minimum-sized structure under a constraint such as on the diameter of the drive pin. One end of the stationary grooved cam is determined from the initial rotational angle $\theta_{0}$ of the lever and the initial drive-side lever length $S_{d, 0}$. The other end is determined from the width of the grooved cams $W$ and the driven- 
side maximum displacement $X_{d n, n}$. Ten control points between the ends of the stationary grooved cam are prepared. Compared with the initial intersection angle, the optimized intersection angle is 25 degrees, and it does not satisfy the criterion. To increase the intersection angle, $\theta_{0}$ should be smaller and $W$ should be wider in order to meet the requirement of inclining the stationary grooved cam curve steeply against the $\mathrm{x}$-axis (Case II). Figure 15 shows the convergence process. The intersection angle after convergence is 40 degrees, much larger than the criterion, and the curvature radii are larger than the initial ones. The contact forces acting on the contact positions between the grooved cams and the drive pin are sufficiently small.

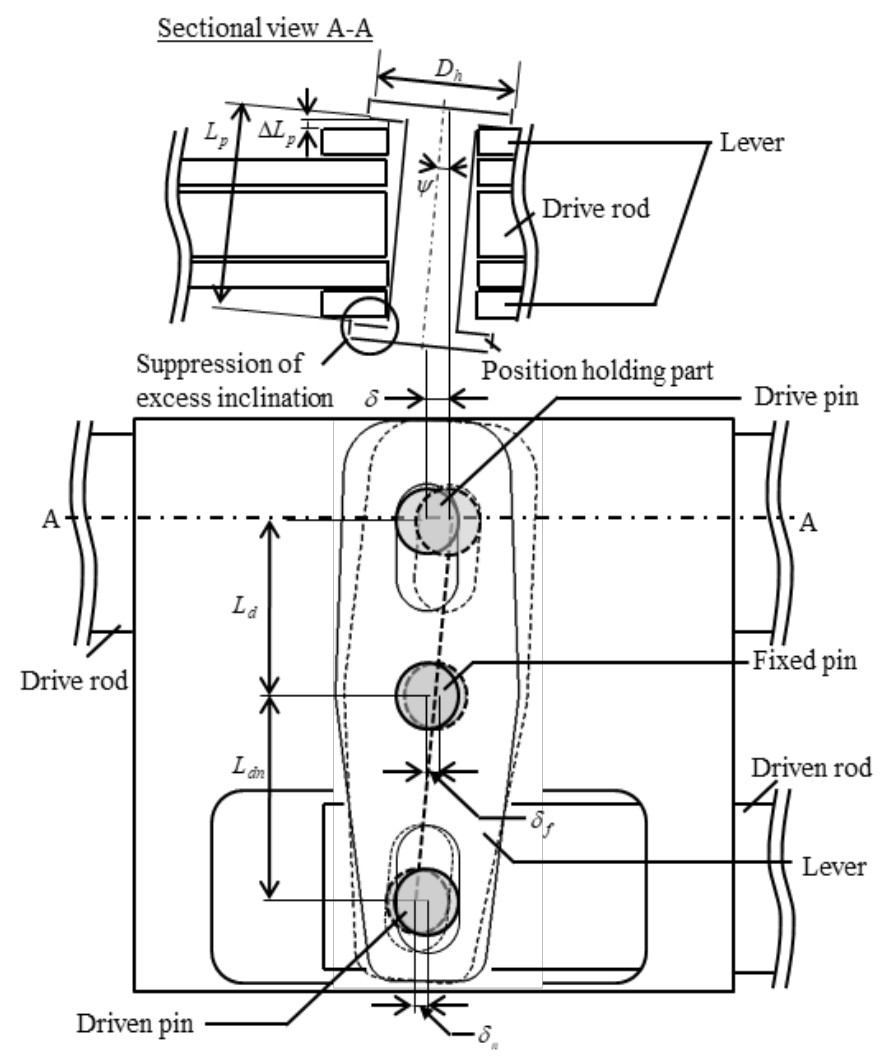

Figure 14: Schematic drawing of the double grooved cams

Table 1: Design results

\begin{tabular}{c|c|c|c|c|}
\hline \multirow{2}{*}{ Dimensions } & \multicolumn{4}{|c}{ Values } \\
\cline { 2 - 5 } & \multicolumn{2}{|c|}{ Case I } & \multicolumn{2}{c}{ Case II } \\
\cline { 2 - 5 } & Init. & Opt. & Init. & Opt. \\
\hline $\begin{array}{c}\text { Lever ratio of driven-side length } \\
\text { to drive-side length }\end{array}$ & \multicolumn{2}{|c}{1} & \multicolumn{2}{|c}{1.2} \\
\hline $\begin{array}{c}|c| \\
\text { Initial angle of ling lever (degrees) }\end{array}$ & \multicolumn{2}{|c|}{45} & \multicolumn{2}{|c}{28.5} \\
\hline Width of grooved cam (a.u.) & \multicolumn{2}{|c|}{1} & \multicolumn{2}{|c}{1.36} \\
\hline $\begin{array}{c}\text { Minimum intersection angle } \\
\text { (degrees) }\end{array}$ & 11 & 25 & 21 & 40 \\
\hline $\begin{array}{c}\text { Minimum curvature radius in } \\
\text { movable grooved cam (mm) }\end{array}$ & 1 & 4 & 17 & 29 \\
\hline $\begin{array}{c}\text { Minimum curvature radius in } \\
\text { stationary grooved cam (mm) }\end{array}$ & 2 & 2 & 7 & 13 \\
\hline
\end{tabular}

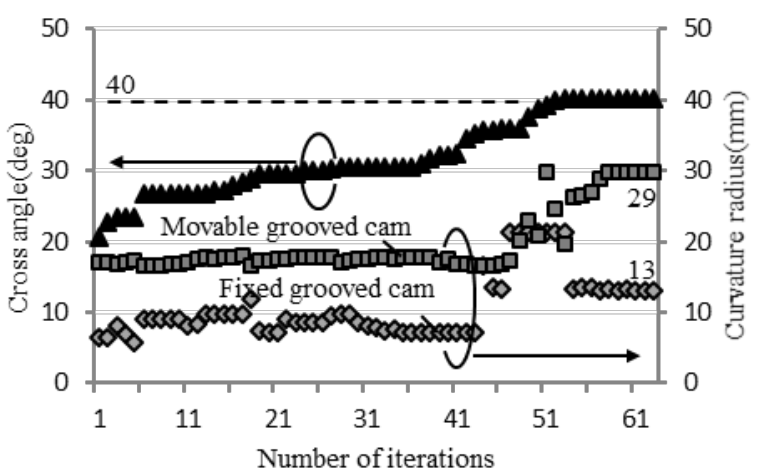

Figure 15: Convergence process

\subsection{Design of pin holding parts}

Figure 16 shows the relationship between the diameter of the pin position holding part and the drive-pin inclination expressed by Equation (19). As the threshold of the drive-pin inclination $\delta_{t}$ is 0.0033 due to the fit tolerances of the fixed pin and the driven pin, the diameter of the position holding part is 0.157 , i.e., 1.96 times larger than the drive pin.

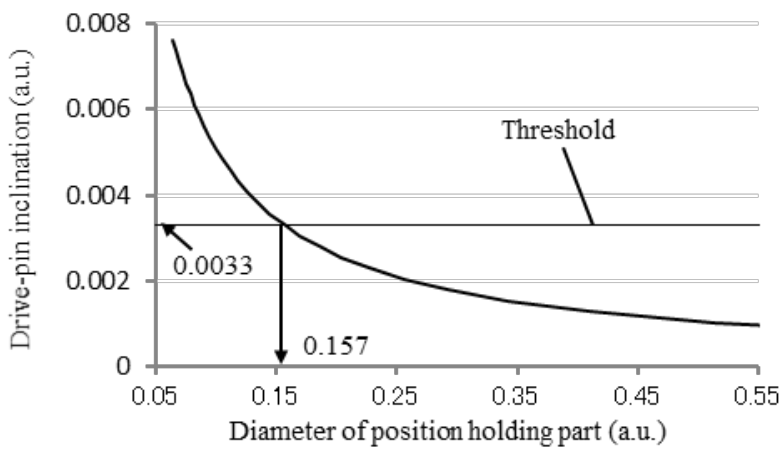

Figure 16: Drive-pin inclination versus diameter of pin position holding part

\section{Motion verification of double grooved cams}

Motion and endurance tests were performed to measure the mechanical reliability of the designed dual motion mechanism.

\subsection{Experimental setup}

Figure 17 shows the experimental setup for the motion test. An interrupter unit is set vertically. The dual motion mechanism is assembled on the top of the interrupter unit. The operating device drives the drive-side moving parts of the interrupter unit vertically. When the drive-side moving parts move downwards, i.e., in an opening motion, the driven-side moving parts move upwards. It should be noted that the direction of the downward motion is the positive direction. The drive-side maximum displacement and driven-side maximum displacement are $150 \mathrm{~mm}$ and $50 \mathrm{~mm}$, respectively. The operating device is a combination of a $4.4-\mathrm{kW}$ servomotor and a link mechanism. The maximum speed is about half of the real-scaled high-voltage circuit breaker. The upward moving speed for returning to the initial position of the opening motion is much slower than the downward moving speed. Linear 
potentiometers are set on the top of the dual motion mechanism to measure the drive-side displacement and the driven-side displacement. A strain gauge is attached on the guide plate to measure strain near the fixed pin where the maximum load acts.

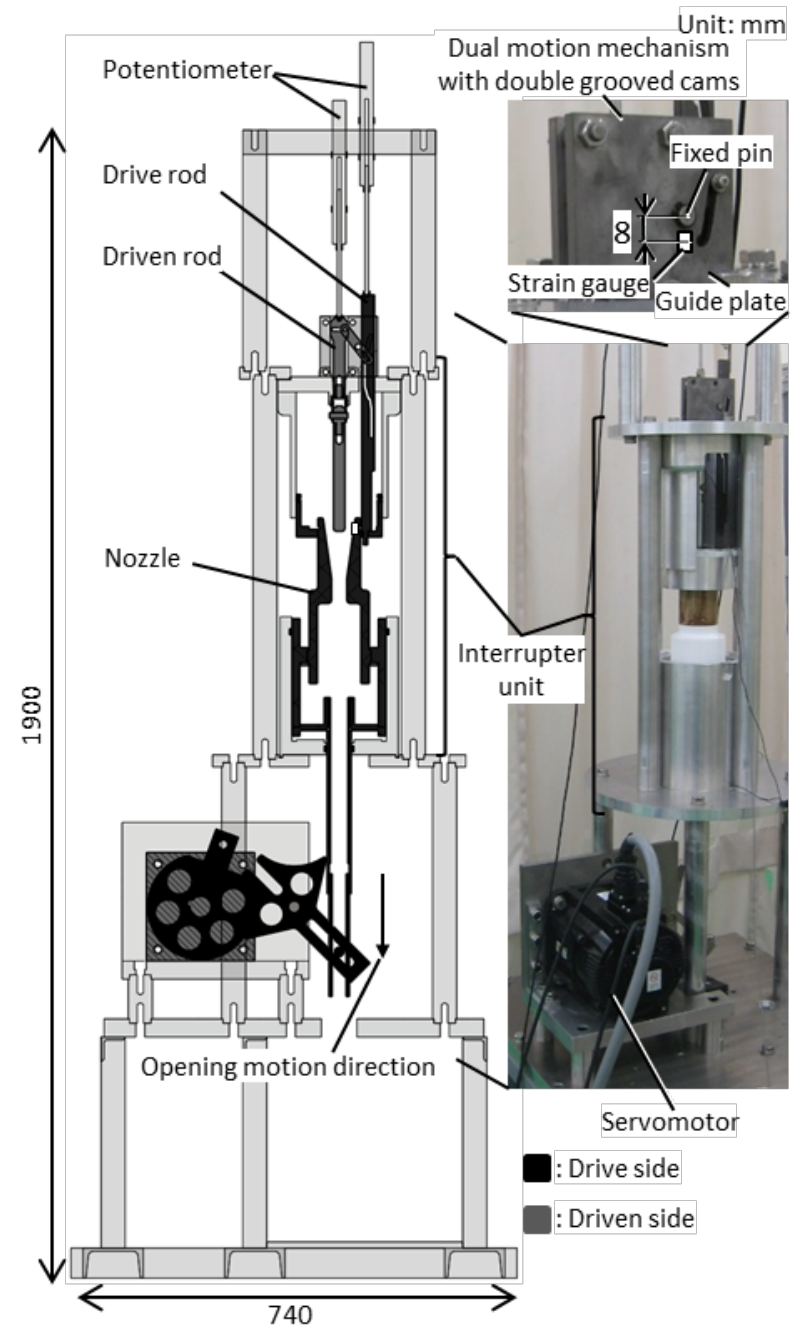

Figure 17: Experimental setup of motion test

\subsection{Motion test results}

Figure 18 compares the measured and calculated displacement curves. The results indicate that the displacement curve is in good agreement with the ideal displacement curve from $0 \mathrm{~mm}$ to -30 $\mathrm{mm}$ on the driven-side. In this period, the drive pin moves smoothly. After that, the deviation from the ideal curve is large, especially around the end of the driven-side motion. This is because the drive pin or the driven pin bends with elastic deformation due to inertia during the driven-side motion. Figure 19 shows the measured strain. Compressive strain occurred due to the reaction force at the start of operation on the driven-side. In the final stage of the operation on the driven-side, tensile strain occurs as a result of the upward force by inertia, and then compressive strain occurs as a result of downward force by gravity. The strains at the beginning and the end of the drive-side operation are almost $0 \%$. This means that there is no residual strain, therefore the movement is not locked and it moves smoothly. Figure 20 shows deviations from the mean value of the maximum compressive strain on the guide plate in a 2,000-motion $\underline{\text { www.astesj.com }}$ test. Since all values fall within the range of $5 \%$, the operation is stable and the dual motion mechanism with the double grooved cams is mechanically reliable.

In addition, an endurance test was performed on a full-scale prototype of the high-voltage circuit breaker. The prototype successfully passed a 10,000-motion test.

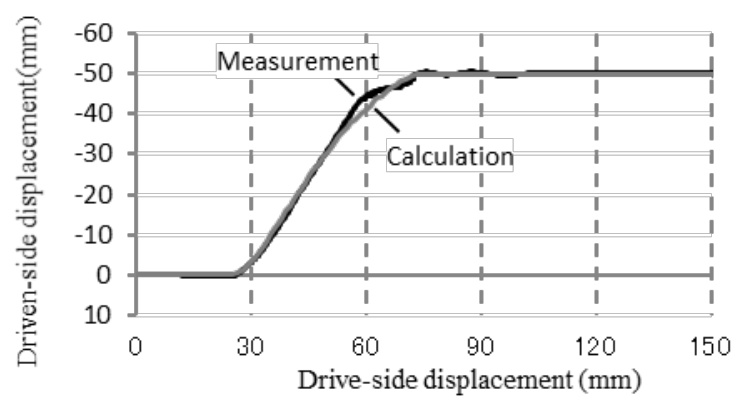

Figure 18: Comparison of measured and designed displacements

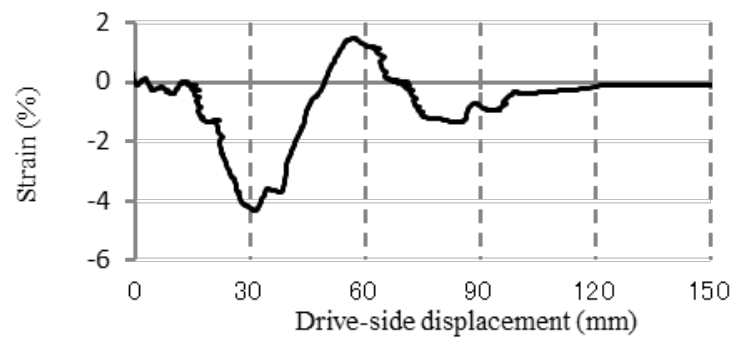

Figure 19: Tensile and compressive stress on the guide plate

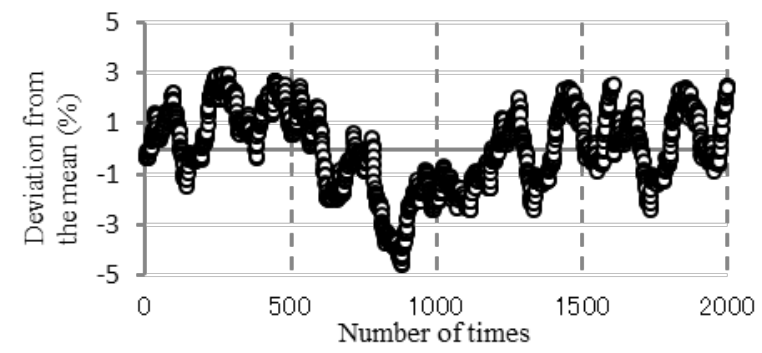

Figure 20: Deviation from the mean value of maximum compressive stress

\section{Conclusion}

The dual motion mechanism with double grooved cams that cross each other was proposed. A pin positioned at the intersection point of the grooved cams rotates a lever linked to both contacts while changing the lever ratio that shortens the path length of the pin movement. The proposed mechanism has three advantages. The first one is that an arbitrarily shaped displacement curve can be expressed. The second one is that the driving force of the pin can be converted efficiently into rotational force of the lever. The third one is that the overall size of the dual motion mechanism can be reduced. The following conclusions were obtained about: optimization of a displacement curve for the high-voltage circuit breaker, the shape of the grooved cams, and the mounting structure for the dual motion mechanism with double grooved cams.

- A displacement curve of the driven-side contact on the opposite side of the drive-side contact linked to the operating mechanism for the high-voltage circuit breaker was optimized, 


\section{Terada et al. / Advances in Science, Technology and Engineering Systems Journal 5, No. 4, 109-118 (2020)}

as the driven-side contact operates quickly only during the capacitive current switching (CCS) period and starts to move before contact separation. The displacement curve can minimize the operating energy and mechanical load acting on the mechanism while keeping the CCS performance high.

- The optimal shape of the double grooved cams designed using the direct search algorithm can minimize the local contact forces acting on the contact positions between the grooved cams and the pin. Also, a position holding part designed in accordance with the gaps between the grooved cams and the pin can maintain the stability of the pin in motion.

- The measured displacement curve was in good agreement with the calculated displacement curve. In addition, since the variation of each measured strain in the 2,000-motion test is small, the operation is stable and the mechanism has high mechanical reliability. Furthermore, a full-scale prototype of the high-voltage circuit breaker successfully passed the 10,000-motion test.

\section{References}

[1] M. Terada, Y. Nakai, H. Hashimoto, D. Ebisawa, H. Urai, Y. Yokomizu, "Design of Dual Motion Mechanism Moving along Optimized Stroke Curve to Improve Capacitive Current Switching Performance for Gas Circuit Breaker" in 2019 5th International Conference on Electric Power Equipment - Switching Technology (ICEPE-ST), Kitakyushu, Japan, 2019. https://doi.org/10.1109/ICEPE-ST.2019.8928703

[2] A. Ahmethodzic, R. P. P. Smeets, V. Kertesz, M. Kapetanovic, K. Sokolija, "Design Improvement of a 245-kV SF6 Circuit Breaker With Double-Speed Mechanism Through Current Zero Analysis," IEEE Transaction on Power Delivery, 25(4), October, 2010. https://doi.org/10.1109/TPWRD.2010.2057262

[3] O. Hunger, "Circuit breaker with a gear having a dead point," U.S. Patent 8415578, 2013.

[4] H. Dienemann, V. Lehmann, H. Marin, "High Voltage Circuit Breaker with Two Arcing Contacts Which Can Be Actuated in an Opposite Direction," U.S. Patent 6271494, 2001.

[5] J. Y. Trepanier, M. Reggio, Y. Lauze, R. Jeanjean, "Analysis of the Dielectric Strength of an SF6 Circuit Breaker," IEEE Transactions on Power Delivery, 6, No. 2, April, 1991. https://doi.org/10.1016/j.aasri.2014.05.029

[6] H. Joshi, A. Pandharker, G. Patil, "Optimization of High Voltage Arc Assist Interrupters," International Journal of Scientific \& Engineering Research, 4(3), March, 2013. https://doi.org/10.1109/TPWRD.2003.817536

[7] Q. Zhang, J. Liu, J. D. Yan, "Flow Structure Near Downstream Electrode of a Gas-Blast Circuit Breaker," IEEE Transactions on Plasma Science, 42(10), October, 2014. https://doi.org/10.1109/TPS.2014.2309174

[8] J.D. Mantilla, C.M. Franck, M. Seeger, "Measurements and Simulations of Cold Gas Flows in High Voltage Gas Circuit Breakers Geometries," Electrical Insulation 2008. ISEI 2008. Conference Record of the 2008 IEEE International Symposium, 720-723, 2008. https://doi.org/10.1109/ELINSL.2008.4570431

[9] H. K. Kim, K. Y. Park, C. H. Im, H. K. Jung, ”Optimal Design of Gas Circuit Breaker for Increasing the Small Current Interruption Capacity," IEEE Transactions on Magnetics, 39(3), May, 2003. https://doi.org/10.1109/TMAG.2003.810176

[10] H. K. Kim, J. K. Chong, K. Y. Park, "Approximation Model-Assisted Optimization Technique to Improve Capacitive Current Interrupting Performance of Gas Circuit Breaker," IEEE Transactions on Magnetics, 45, No. 3, March, 2009. https://doi.org/10.1109/TMAG.2009.2012746

[11] M. T. Dhotre, X. Ye, F. Linares, P. Skarby, S. Kotilainen, "Multiobjective Optimization and CFD Simulation for a High-Voltage Circuit Breaker," IEEE Transaction on Power Delivery, 27(4), October, 2012. https://doi.org/10.1109/TPWRD.2012.2207133

[12] N. Yamada, S. Taki, N. Osawa, Y. Yoshioka, "Investigation of an Automatic Design Method of the Optimum GCB Insulating Structure and Necessary Opening Speeds for Successful Capacitive Current Switching," Proceeding of the ICEE 2004, Sapporo, Japan, 7(2), 901-906, 2004. https://www.researchgate.net/publication/267371487

[13] S. Delic, D. Beslija, D. Gorenc, A. Hajdarovic, M. Kapetanovic, "Capacitive current breaking capability estimation for a $145 \mathrm{kV} 40 \mathrm{kA}$ GIS circuit breaker," 2015 3rd International Conference on Electric Power Equipment - Switching $\begin{array}{llll}\text { Technology } & \text { (ICEPE-ST), October } & 25-28, & 2015 .\end{array}$ https://doi.org/10.1109/ICEPE-ST.2015.7368412
[14] M. Kang, K. H. Kim, T. Ohk, Y. T. Yoon, "Analysis of Insulation Coordination for Dual Motion Mechanism Gas Circuit Breaker," 3rd International conference on Electric Power Equipment - Switching Technology (ICEPE-ST), 2015. https://doi.org/10.1109/ICEPEST.2015.7368319

[15] D. M. Tsay, C. O. Huey, "Cam Motion Synthesis Using Spline Functions," Trans. ASME, Journal of Mechanisms, Transmissions, and Automation in Design, 110(2), 161-165, June, 1988. https://doi.org/10.1115/1.3258921

[16] J. S. Jang, J. J. Lee, J. H. Sohn, H. W. Kim, B. T. Bae, W. S. Yoo, "A Cam Profile Design of a Circuit Breaker by Using Multibody Dynamics Analysis," 2015 3rd International Conference on Electric Power Equipment - Switching Technology (ICEPE-ST), October 25-28, 2015. https://doi.org/10.1109/ICEPE-ST.2015.7368440

[17] A. Pourghodrat, C.A. Nelson, "A case study of designing a cam-follower mechanism with cycloidal motion," 13th World Congress in Mechanism and Machine $\quad 2011 . \quad$ Science, 2 ttps://www.dmglib.org/dmglib/handler?docum $=22419009$

[18] E. Catmull, R. Rom, A class of local interpolating splines, Computer Aided Geometric Design, Academic Press, New York, 317-326, 1974.

[19] F. Endo, M. Sato, M. Tsukushi, Y. Yoshioka, K. Saito, K. Hirasawa, "Analytical prediction of transient breakdown characteristics of $\mathrm{SF}_{6}$ gas circuit breakers," IEEE Transaction on Power Delivery, 4(3), July, 1989. https://doi.org/10.1109/MPER.1989.4310811

[20] D. M. Himmelblau, Applied Nonlinear Programming, McGraw Hill, New York, 1972. 